\title{
Multivessel percutaneous coronary intervention in acute myocardial infarction complicated by cardiogenic shock - a case report
}

\section{Željko Baričević ${ }^{*}$}

University Hospital Centre Zagreb, Zagreb, Croatia

\begin{abstract}
KEYWORDS: acute myocardial infarction, cardiogenic shock, percutaneous coronary intervention, multivessel disease.

CITATION: Cardiol Croat. 2018;13(1-2):23. | https://doi.org/10.15836/ccar2018.23

*ADDRESS FOR CORRESPONDENCE: Željko Baričević, Klinički bolnički centar Zagreb, Kišpatićeva 12, HR-10000 Zagreb, Croatia. / Phone: +385-1-2367-508 / E-mail: zbaricev@gmail.com

ORCID: Željko Baričević, https://orcid.org/0000-0002-5420-2324

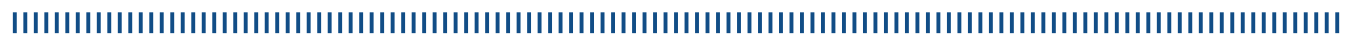

Background: Acute myocardial infarction complicated by cardiogenic shock is associated with high early mortality of 40 to $50 \%$, with many of these patients known to have multivessel coronary artery disease. Although current guidelines recommend multivessel revascularization if feasible ${ }^{1}$, the findings of the recent CULPRIT-SHOCK trial show that non-culprit vessel percutaneous coronary intervention (PCI) in this setting increases all-cause mortality ${ }^{2}$. However, the clinical scenarios may differ significantly and there is still room to debate whether a straightforward infarction-related artery PCI only is the correct approach in every single case.
\end{abstract}

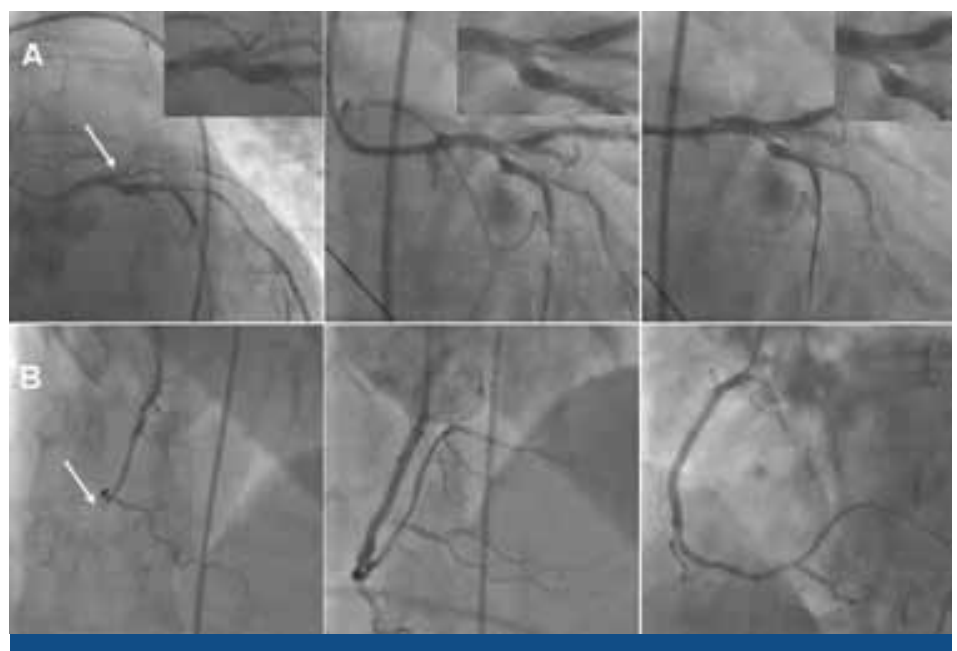

FIGURE 1. Multivessel percutaneous coronary intervention protocol. Provisional left main coronary artery/left anterior descending artery stenting (A) followed by revascularization of the chronically occluded mid-right coronary artery (B).

Case report: A 50-year-old male non-smoker with history of arterial hypertension was admitted to the hospital complaining of upper abdominal pain radiating toward the neck that had begun 5 hours before, followed by general weakness, malaise and somnolence. At the time of admission cardiogenic shock was noted with a blood pressure of $60 / 40 \mathrm{mmHg}$ and ECG revealing sinus bradycardia of $45 / \mathrm{min}$ with right bundle branch block. Bedside echocardiogram showed severely reduced left ventricular systolic function (LVEF 20\%) with hypokinesia of anteroseptal, apical, anterior and inferior wall segments, together with severely hypokinetic right ventricle. Urgent coronary angiography demonstrated acute thrombotic occlusion of the ostial left anterior descending artery (LAD) and short chronic total occlusion (CTO) of the mid-right coronary artery (RCA) segment. Due to profound shock despite high-dose inotropic and vasopressor therapy, the percutaneous peripheral veno-arterial extracorporeal membrane oxygenation (VA-ECMO) support was initiated. A successful provisional stenting of the left main coronary artery and LAD using 2 drug eluting stents was then performed, followed by the LAD/circumflex artery bifurcation optimization using standard proximal optimization technique (POT), side-branch inflation, kissing balloons inflation and re-POT sequence (Figure 1A). Being aware of the large amount of affected myocardium in a latecomer with uncertain potential for recovery and expected detrimental hemodynamic side effects of the prolonged VA-ECMO support, an ad hoc anterograde PCI CTO RCA was done to recruit myocardial contractility reserve to help promote early weaning from ECMO (Figure 1B). The mechanical support was successfully removed 8 days after the implantation, with full RV recovery noted, coupled with LVEF increase to $35 \%$ due to inferior wall contractility restitution, but showing no improvement in the LAD territory. The patient was transferred to rehabilitation facility with uneventful course

RECEIVED:

February 3, 2018

ACCEPTED:

February 10, 2018

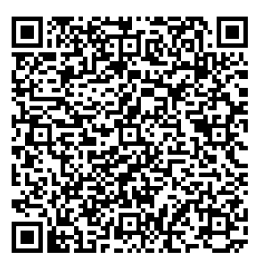

Conclusion: Immediate non-culprit vessel PCI may be considered in certain patients with acute myocardial infarction complicated by cardiogenic shock, especially with ongoing mechanical circulatory support counterbalancing potential drawbacks of prolonged intervention.

LITERATURE IIIIIIIIIIIIIIIIIIIIIIIIIIIIIIIIIIIIIIIIIIIIIIIIIIIIIIIIIIIIIIIIIIIIIIIIIIIIIIIIIIIIIIIIIIIIIIIIIIIIIIII

1. Ibanez B, James S, Agewall S, Antunes MJ, Bucciarelli-Ducci C, Bueno H, et al; ESC Scientific Document Group. 2017 ESC Guidelines for the management of acute myocardial infarction in patients presenting with ST-segment elevation: The Task Force for the management of acute myocardial infarction in patients presenting with ST-segment elevation of the European Society of Cardiology (ESC). Eur Heart J. $2018 \mathrm{Jan}$ 7;39(2):119-177. https://doi.org/10.1093/eurheartj/ehx393

2. Thiele H, Akin I, Sandri M, Fuernau G, de Waha S, Meyer-Saraei R, et al; CULPRIT-SHOCK Investigators. PCI strategies in patients with acute myocardial infarction and cardiogenic shock. N Engl J Med. 2017 Dec 21;377(25):2419-2432. https://doi.org/10.1056/NEJMoal710261 